\title{
Modelado y simulación CFD de un sistema de refrigeración para el almacenamiento de vacunas contra la Influenza Estacional
}

\section{Modeling and CFD simulation of a cooling system for the storage of vaccines against Seasonal Flu}

VALLE-HERNÁNDEZ, Julio†*, DÍAZ-MONROY, Joanna, LOZANO-OLMEDO, Britania Junery y ROJAS-ÁVILA, Apolo

Universidad Autónoma del Estado de Hidalgo. Escuela Superior de Apan

Universidad Politécnica Metropolitana de Hidalgo. Maestría en Ingeniería Aeroespacial

ID 1 ${ }^{\text {er }}$ Autor: Julio, Valle-Hernández / ORC ID: 0000-0001-8957-0066, Researcher ID Thomson: O-7339-2018, CVU CONACYT ID: 210743

ID $1^{\text {er }}$ Coautor: Joanna, Díaz-Monroy / ORC ID: 0000-0002-2720-2978

ID $2^{\text {do }}$ Coautor: Britania Junery, Lozano-Olmedo / ORC ID: 0000-0002-7808-4609

ID $3^{\text {er }}$ Coautor: Apolo, Rojas-Ávila / ORC ID: 0000-0003-2934-2533, Researcher ID Thomson: O-4655-2018, CVU CONACYT ID: 905177

\section{Resumen}

Es muy importante realizar campañas de vacunación en las zonas marginadas de nuestro país, debido a que los habitantes de estas regiones están más vulnerables a contraer enfermedades, pero para poder transportar las vacunas se necesitan contenedores adecuados para que éstas lleguen con sus propiedades y puedan proteger a cada persona. La vacuna contra la Influenza Estacional es transportada en neveras portátiles a temperaturas de entre 2 y $8{ }^{\circ} \mathrm{C}$, y el tiempo de conservación está dentro de los 3 y 7 días para el primer caso y de 12 a 36 horas para el segundo. En este trabajo se presenta el modelado y simulación mediante Dinámica de Fluidos Computacionales (CFD) de un sistema refrigerado, a partir de celdas termoeléctricas, para el almacenamiento de vacunas contra la influenza. En los resultados se muestra la distribución de temperatura dentro del sistema refrigerado y la transferencia de calor en cada elemento contenedor, así como el arreglo más adecuado para mantener cada vacuna a la temperatura óptima de almacenamiento. Poder contar con sistemas portátiles que permitan el traslado óptimo de vacunas manteniéndolas refrigeradas de forma constante tendría un impacto positivo en la salud de las personas que radican en zonas marginadas.

Sistema de refrigeración termoeléctrico, Simulación CFD, Vacunas contra influenza estacional

\begin{abstract}
It is very important to carry out vaccination campaigns in the marginalized areas of our country, because they are more vulnerable to contracting diseases, but to do the appropriate containers are needed so that they arrive with their properties and to protect each person. The Seasonal Influenza vaccine is transported in portable refrigerators at a temperature of 2 and $8^{\circ} \mathrm{C}$, and the storage time is within 3 and 7 days for the first and 12 to 36 hours for the second. This paper presents the modeling and simulation by Computational Fluid Dynamics (CFD) of a refrigerated system, from thermoelectric cells, for the storage of influenza vaccines. The results show the temperature distribution within the refrigerated system and the transfer of heat in each container element, as well as the most appropriate arrangement to keep each vaccine to the optimal storage temperature. Being able to count on portable systems that allow the optimal transfer of vaccines keeping refrigerated in a constant way would have a positive impact on the health of people who live in marginalized areas.
\end{abstract}

Thermoelectric cooling system, Simulation CFD, Vaccines against seasonal flu

Citación: VALLE-HERNÁNDEZ, Julio, DÍAZ-MONROY, Joanna, LOZANO-OLMEDO, Britania Junery y ROJASÁVILA, Apolo. Modelado y simulación CFD de un sistema de refrigeración para el almacenamiento de vacunas contra la Influenza Estacional. Revista de Simulación y Laboratorio. 2019, 6-21: 12-18

*Correspondencia al Autor (Correo electrónico: julio_valle@uaeh.edu.mx)

$\dagger$ Investigador contribuyendo como primer Autor 


\section{Introducción}

Dentro del mercado de cámaras frigoríficas encontramos diversos tipos de contenedores para el transporte de vacunas, entre ellos encontramos las neveras portátiles que tienen la capacidad de conservar las vacunas de entre 3 y 7 días, y los porta vacunas los cuales tienen una capacidad de enfriamiento que oscila entre 12 y 36 horas, estos dos contenedores son utilizados para el transporte de pocas unidades en trayectos cortos y para que tengan un buen funcionamiento es recomendable que se abran lo menos posible (Álvarez, 2019).

La refrigeración termoeléctrica está basada en el efecto Peltier, y constituye un sistema de conversión directa de electricidad en frío, sin pasar por energía mecánica ni térmica como ocurre con los sistemas convencionales de compresión o absorción (Villarrubia, 2001). Al tratarse de un sistema completamente estático, sin partes móviles, y alimentado únicamente por corriente continua, es muy útil en todas aquellas aplicaciones en donde la carga a refrigerar esté en movimiento (Villarrubia, 2001), como es el caso de las campañas de vacunación.

El presente trabajo muestra el modelado y simulación, por medio de la Dinámica de Fluidos Computacionales (CFD), de un sistema refrigerado termoeléctricamente para el almacenamiento de vacunas. El diseño del contenedor propuesto buscó reducir al máximo las cargas térmicas generadas, para mantener las temperaturas de operación un mayor tiempo y reducir su consumo energético, el cual se genera mediante energía solar. La simulación CFD nos permitió observar la distribución de temperatura dentro del contenedor, para estimar la transferencia de calor en cada vacuna contenida, y se propuso el arreglo más adecuado para mantener las vacunas en condiciones óptimas hasta su aplicación.

A partir del análisis de la simulación CFD se determinó la viabilidad del sistema, permitiendo representar diversos escenarios mediante la simulación, evaluación, estudio y análisis de sustancias y materiales en diferentes ambientes.

\section{Metodología}

La metodología a seguir inició con la descripción del sistema de refrigeración termoeléctrico propuesto, cuyo diseño se presenta en otro trabajo (Valle, et al, 2019). En este trabajo se presentó el desarrollo del modelado y simulación del sistema propuesto, y se analizaron los resultados del mismo.

El modelo matemático se fundamenta en las ecuaciones de transferencia de calor, a partir de las dimensiones, geometría, materiales, y condiciones de operación del sistema.

Una vez realizado el paso anterior, se procedió a obtener la solución numérica de las ecuaciones que gobiernan el problema, mediante simulación CFD, y se analizaron los resultados obtenidos determinando su viabilidad.

\section{Desarrollo}

\section{Descripción del sistema}

El contenedor de vacunas que proponen Valle et al (2019), es un dispositivo óptimo que reduce las cargas térmicas generadas por transmisión de paredes y por infiltraciones de aire exterior. Lo anterior se consigió reduciendo al mínimo el área superficial de transferencia de calor, por su diseño cilíndrico, así como por un sistema dispensador de vacunas. El proceso de refrigeración se realizó mediante celdas Peltier y es alimentado por medio de un panel fotovoltaico de $80 \mathrm{~W}$ que asegura una temperatura estable para las vacunas.

El contenedor cilíndrico tiene un volumen de $3 \mathrm{~L}$, con una altura de $0.226 \mathrm{~m}$ y un radio de $0.065 \mathrm{~m}$. Estas dimensiones otorgan la menor área de transferencia de calor para el volumen dado.

El material con el que se fabricó el contenedor es de plástico de alta densidad resistente al impacto, con una tapa sólida sellada herméticamente. El contenedor se recubrió con un aislante térmico de $20 \mathrm{~mm}$ de poliuretano de alta densidad libre de CFC's. 


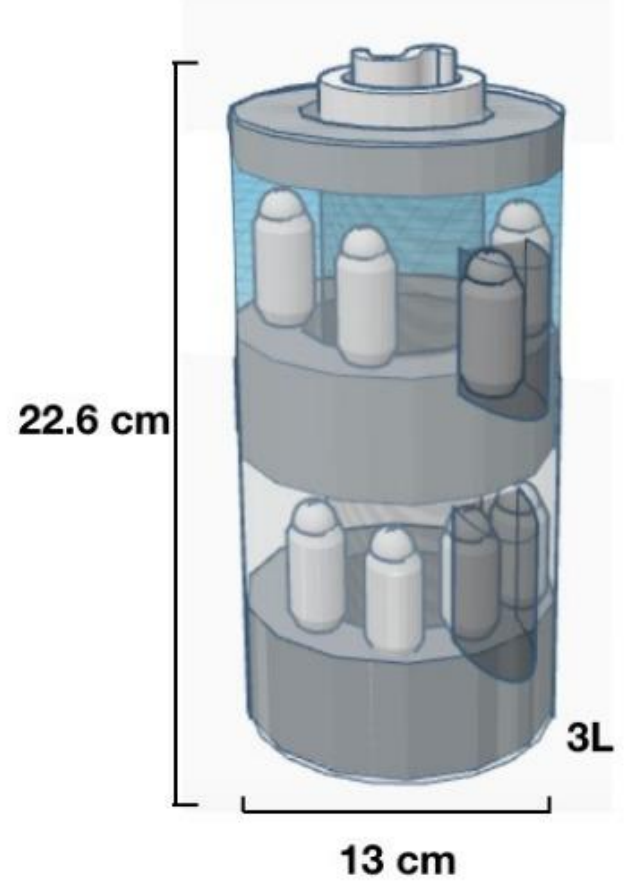

Figura 1 Diseño y dimensiones del contenedor de vacunas Fuente: Elaboración Propia

En la Figura 1 se puede observar el diseño del contenedor para transportar las vacunas de forma refrigerada. El sistema tiene la capacidad de refrigerar $100 \mathrm{ml}$ de vacunas que están contenidas en frascos de $5 \mathrm{ml}$ de capacidad, es decir, 20 frascos.

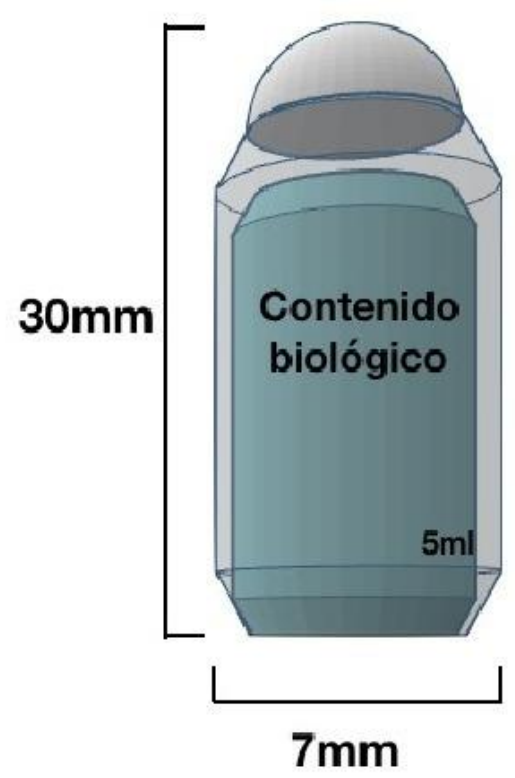

Figura 2 Dimensiones del frasco de una vacuna Fuente: Elaboración Propia

En la Figura 2 se pueden observar las dimensiones del frasco de la vacuna a refrigerar, en este caso de las vacunas contra la influenza AH1N1 cada dosis es de $0.5 \mathrm{ml}$.

\section{Condiciones de operación}

El contenedor de vacunas se expuso a una temperatura máxima externa de $35{ }^{\circ} \mathrm{C}$ y una carga térmica total de $10.7 \mathrm{~W}$, la temperatura interna fue de entre $2{ }^{\circ} \mathrm{C}$ y $8{ }^{\circ} \mathrm{C}$ que es la temperatura a la que se conservan las vacunas. (Valle, et al, 2019).

\section{Modelado Matemático}

El modelado matemático se realizó a partir del balance de energía a partir de la ecuación general de transferencia de calor y de la ecuación de enfriamiento de Newton, para lo cual fue necesario calcular el coeficiente convectivo interno y externo.

\section{Ecuación general de transferencia de calor}

$\rho C_{p} \frac{\partial T}{\partial t}+\rho C_{p} u \cdot \nabla T+\nabla \cdot q=Q$

Donde:

$q=-k \nabla T$

$T:$ Temperatura.

$\rho$ : Densidad del aire.

$C_{p}$ : Capacidad calorífica del aire.

$u$ : Campo de velocidades del aire interno.

$Q$ : Sumidero de calor.

Flujo de calor convectivo

$q=h\left(T_{e x t}-T\right)$

Donde:

$h$ : Coeficiente convectivo.

$T_{a m b}$ : Temperatura del ambiente.

$T$ : Temperatura de la pared.

\section{Cálculo del coeficiente convectivo interno}

\section{- $\quad$ Velocidad del aire}

$V_{\text {aire }}=Q_{\text {vent }} * \frac{1}{A_{\text {vent }}}$

Donde:

$V_{\text {aire }}=$ Velocidad del aire $\left[\frac{m}{s}\right]$

$Q_{\text {vent }}=$ Flujo volumétrico de aire entregado por el ventilador $\left[\frac{m^{3}}{s}\right]$

$A_{\text {vent }}=$ Área del ventilador $\left[\mathrm{m}^{2}\right]$ 


\section{- Diámetro hidráulico}

$D h_{\text {int }}=\frac{4 * A_{m}}{P_{m}}$

Donde:

$D h_{\text {int }}=$ Diámetro hidráulico al interior de la cámara $[m]$

$A_{m}=$ Área mojada $\left[\mathrm{m}^{2}\right]$

$P_{m}=$ Perímetro mojado $[m]$

\section{- $\quad$ Número de Reynolds}

$R e_{i n t}=\frac{\rho_{\text {int }} * v_{c_{i n t}} * D h_{\text {int }}}{U_{\text {int }}}$

Donde:

$R e_{i n t}=$ Número de Reynolds al interior de la cámara

$\rho_{\text {int }}=$ Densidad del aire en el interior $\left[\frac{\mathrm{kg}}{\mathrm{m}^{3}}\right]$

$v_{c_{\text {int }}}=$ Velocidad del aire en el interior $\left[\frac{m}{s}\right]$

$D h_{\text {int }}=$ Diámetro hidráulico equivalente $[\mathrm{m}]$

$U_{\text {int }}=$ Viscosidad dinámica interior $\left[\frac{\mathrm{Ns}}{\mathrm{m}^{2}}\right]$

\section{- $\quad$ Número de Nusselt}

$N u_{i}=0.023 * \operatorname{Re}_{\text {int }}^{\frac{4}{5}} * \operatorname{Pr}^{n}$

Donde:

$N u_{i}=$ Número de Nusselt interior

$R e_{i n t}=$ Número de Reynolds al interior de la cámara

$\operatorname{Pr}=$ Número de Prandtl

\section{Coeficiente convectivo interno}

$h_{i}=\frac{N u_{i} * k_{i}}{L_{c}}$

Donde:

$h_{i}=$ Coeficiente convectivo interno

$N u_{i}=$ Número de Nusselt interior

$k_{i}=$ Conductividad térmica del aire $\left[\frac{\mathrm{W}}{\mathrm{mK}}\right]$

$L_{c}=$ Longitud característica $[\mathrm{m}]$

En la Tabla 1, se muestran los valores de los números adimensionales para el cálculo del coeficiente convectivo interno.

\begin{tabular}{|l|r|l|}
\hline Parámetro & Valor & Unidades \\
\hline$D h_{\text {int }}$ & 0.13 & $M$ \\
\hline$R e_{\text {int }}$ & 13121 & Adimensional \\
\hline$N u_{i}$ & 54.7 & Adimensional \\
\hline$P r_{i}$ & 1.6 & Adimensional \\
\hline $\boldsymbol{h}_{\boldsymbol{i}}$ & $\mathbf{4 0 . 3}$ & $\boldsymbol{W} / \boldsymbol{m} \boldsymbol{K}$ \\
\hline
\end{tabular}

Tabla 1 Parámetros obtenidos a partir de las ecuaciones (3), (4), (5), (6) у (7)

Fuente: Elaboración Propia

\section{Cálculo del coeficiente convectivo externo}

\section{- $\quad$ Temperatura de Película}

$T_{f e}=\frac{T_{a m b}+T s_{e x t}}{2}$

Donde:

$T_{f e}=$ Temperatura de película al exterior de la cámara $[\mathrm{K}]$

$T_{a m b}=$ Temperatura del aire exterior $[\mathrm{K}]$

$T s_{\text {ext }}=$ Temperatura de la superficie de la pared exterior $[\mathrm{K}]$

\section{- Número de Prandtl para el aire externo}

$\operatorname{Pr}_{e}=\frac{C p_{e} * \mu_{e}}{k_{e}}$

Donde:

$P r_{e}=$ Número de Prandtl para el aire externo

$C p_{e}=$ Calor especifico del aire $[\mathrm{J} / \mathrm{kgK}]$

$\mu_{e}=$ Viscosidad del aire $\left[\mathrm{Pa}^{*} \mathrm{~s}\right]$

$k_{e}=$ Conductividad térmica del aire externo [W/mK]

\section{- Número de Grashof \\ $G r=\frac{g * \beta e *\left(T_{a m b}-T_{f e}\right) * L^{3}}{v_{e}^{2}}$}

Donde:

$G r=$ Número de Grashof

$g=$ Gravedad $\left[\mathrm{m} / \mathrm{s}^{2}\right]$

$\beta e=$ Coeficiente de expansión $\left[\mathrm{K}^{-1}\right]$

$T_{a m b}=$ Temperatura del aire exterior $[\mathrm{K}]$

$T_{f e}=$ Temperatura de película al exterior de la cámara $[\mathrm{K}]$

$L=$ Longitud característica $[\mathrm{m}]$

$v_{e}=$ Viscosidad cinemática del aire exterior $\left[\mathrm{m}^{2} / \mathrm{s}\right]$ 


\section{- $\quad$ Número de Rayleigh}

$R a_{v e}=G r * P r_{e}$

Donde:

$R a_{v e}=$ Número de Rayleigh

$G r=$ Número de Grashof

$P r_{e}=$ Número de Prandtl para el aire externo

\section{Número de Nusselt para pared vertical}

$$
N u_{v e}=0.68+\frac{0.67 * R a_{v e}^{\frac{1}{4}}}{\left[1+\left(\frac{0.492}{P r_{e}}\right)^{\frac{9}{16}}\right]^{\frac{4}{9}}}
$$

Donde:

$N u_{v e}=$ Número de Nusselt para pared vertical $R a_{v e}=$ Número de Rayleigh

$P r_{e}=$ Número de Prandtl para el aire externo

\section{- Número de Nusselt para pared horizontal}

$N u_{h}=0.54 * R a^{\frac{1}{4}}$

Donde:

$N u_{h}=$ Número de Nusselt para pared horizontal $R a=$ Número de Rayleigh (Godoy, 2016)

En la Tabla 2, se muestran los valores de los números adimensionales para el cálculo del coeficiente convectivo externo.

\begin{tabular}{|l|r|l|}
\hline Parámetro & \multicolumn{1}{c|}{ Valor } & Unidades \\
\hline$P r_{e}$ & 0.715 & Adimensional \\
\hline$G r$ & 48278 & Adimensional \\
\hline$R a_{v e}$ & 34518 & Adimensional \\
\hline$N u_{v e}$ & 7.70 & Adimensional \\
\hline$N u_{h}$ & 7.36 & Adimensional \\
\hline $\boldsymbol{h}_{\boldsymbol{e}}$ & $\mathbf{6 . 1 6}$ & $\boldsymbol{W} / \mathbf{m} \boldsymbol{K}$ \\
\hline
\end{tabular}

Tabla 2 Parámetros obtenidos a partir de las ecuaciones (8), (9), (10), (11), (12) y (13).

Fuente: Elaboración Propia

\section{Resultados}

Para realizar la simulación se consideraron las características mostradas en la Tabla 3, a partir de las cuales se obtuvo la distribución de temperaturas al interior del dispositivo que se muestra en los siguientes gráficos.

\begin{tabular}{|l|r|l|}
\hline \multicolumn{1}{|c}{ Característica } & \multicolumn{1}{c|}{ Valor } & \multicolumn{1}{c|}{ Unidades } \\
\hline Coeficiente convectivo externo & 40.3 & $\mathrm{~W} / \mathrm{mK}$ \\
\hline Coeficiente convectivo interno & 6.16 & $\mathrm{~W} / \mathrm{mK}$ \\
\hline Temperatura ambiente & 35 & ${ }^{\circ} \mathrm{C}$ \\
\hline
\end{tabular}

Tabla 3 Parámetros para la simulación del modelo Fuente: Elaboración Propia

Los gráficos 1 y 2 , muestran la distribución axial de temperaturas en el interior del cilindro refrigerado y en la pared interna del contenedor, respectivamente. Se observa que se tiene una temperatura mínima en la cara superior del contenedor, donde esta sujeto el dispositivo termoeléctrico, al ir descendiendo por el contenedor las temperaturas aumentan, pero sin sobrepasar los $8^{\circ} \mathrm{C}$ por lo que la refrigeración para contener las vacunas es la adecuada.

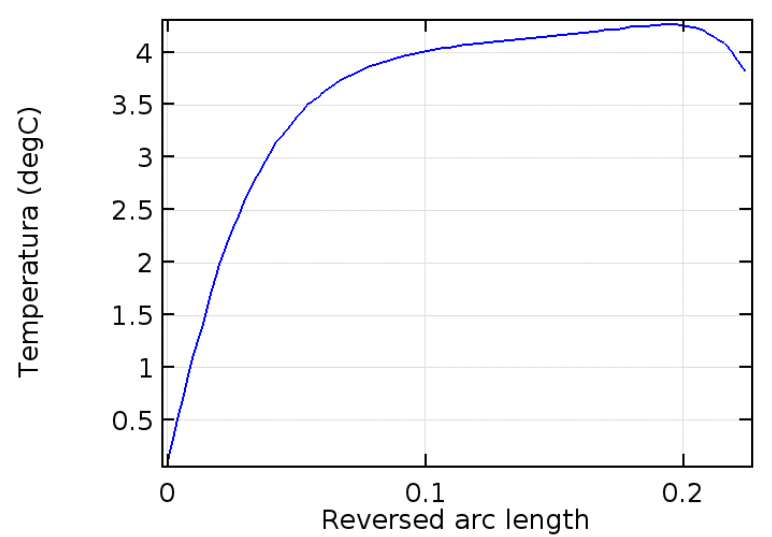

Grafico 1 Distribución axial de temperatura en el interior del contenedor refrigerado

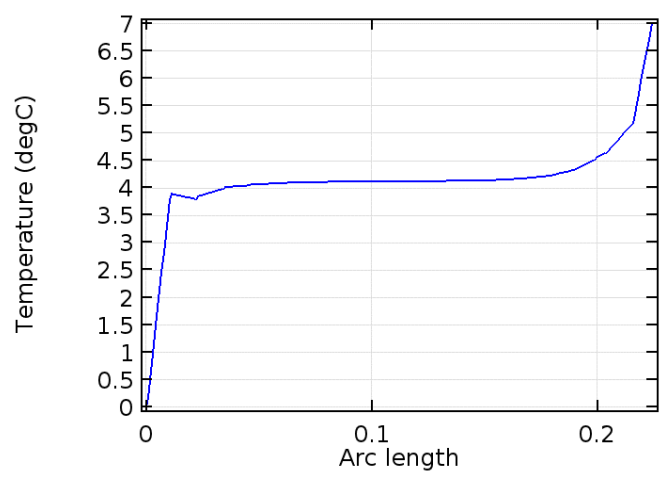

Grafico 2 Distribución axial de temperatura en la cara interna del contenedor refrigerado

En el Grafico 3 se muestran la distribución radial de temperatura. En al centro del cilindro (línea verde), se pudo observar que la temperatura es prácticamente constante en cualquier punto de la circunferencia, con una temperatura de $4.1{ }^{\circ} \mathrm{C}$ aproximadamente, mientras que en los extremos; a $4 \mathrm{~cm}$ de la tapa (línea azul), y a $4 \mathrm{~cm}$ de la base (línea roja), la temperatura aumenta de forma no lineal mientras nos alejamos del centro. 
Cabe mencionar que en cualquiera de los tres casos la temperatura en los extremos no supera $\operatorname{los} 5^{\circ} \mathrm{C}$, por lo que las condiciones térmicas adecuadas para el almacenamiento de las vacunas están garantizadas.

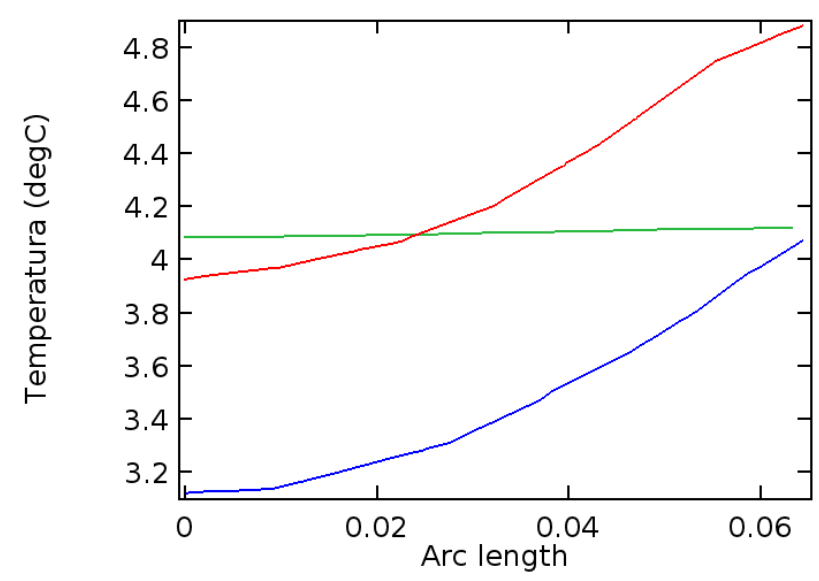

Grafico 3 Distribución radial de temperaturas en el interior del contenedor refrigerado

En la figura 3, se muestra como se distribuye la temperatura dentro del contenedor, y un posible arreglo con dos secciones de almacenamiento de vacunas. La primera sección está entre la tapa y $8 \mathrm{~cm}$ debajo de ésta, mientras que la segunda entre los 12 y $20 \mathrm{~cm}$ de la misma. Bajo este arreglo podemos garantizar que las vacunas se encuentran entre $2^{\circ} \mathrm{C}$ y $4^{\circ} \mathrm{C}$ de temperatura.

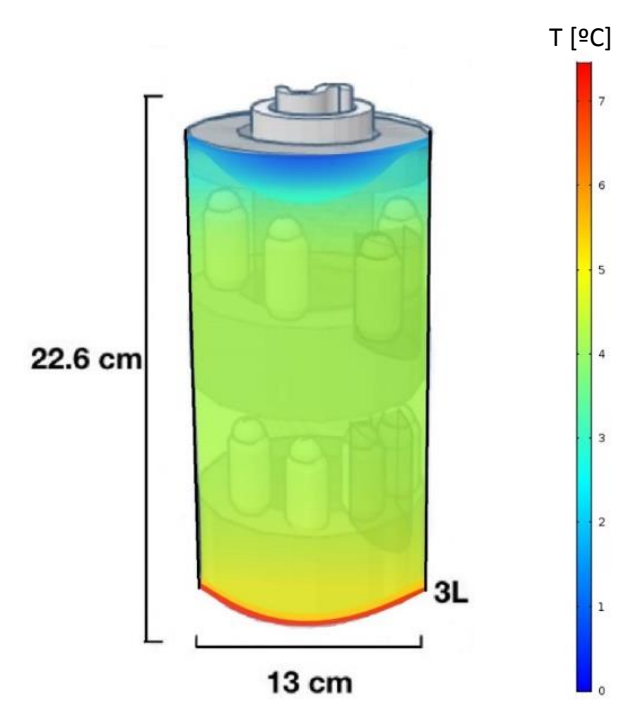

Figura 3 Distribución de temperaturas en el contenedor refrigerado.

Fuente: Elaboración Propia

\section{Conclusiones}

A partir de la distribución de temperaturas en el dispositivo refrigerado para el traslado de vacunas, se pudo concluir que el diseño es viable energéticamente hablando.
Además, se pudo observar que las vacunas que requieran un tiempo de almacenamiento de alrededor de 3 a 6 días se deben colocar en el primer contenedor a $4 \mathrm{~cm}$ de la tapa. $\mathrm{La}$ conservación de las vacunas en el segundo contenedor, en la sección que va de los $13 \mathrm{~cm}$ a los $16 \mathrm{~cm}$ desde la tapa, es de alrededor de 4 días ya que se tiene una temperatura promedio de $3.5^{\circ} \mathrm{C}$.

Cabe mencionar que, aunque en este trabajo se presenta el arreglo descrito en los resultados, las secciones de acomodo pueden variar de acuerdo al número de vacunas que se deseen almacenar, pero el rango de temperaturas requerido $\left(2^{\circ} \mathrm{C}\right.$ a $\left.8^{\circ} \mathrm{C}\right)$ siempre se garantiza.

No obstante, para viajes de más de 2 días, se debe evitar la colocación de las vacunas en la sección comprendida entre los 18 a los $22 \mathrm{~cm}$ ya que se encuentran temperaturas de más de $4^{\circ} \mathrm{C}$ que reducen el tiempo de conservación de las mismas.

Las condiciones de operación a las cuales se simuló el dispositivo fueron las extremas, con una temperatura en el exterior de $35^{\circ} \mathrm{C}$ y considerando la mayor carga térmica, las cuales se tienen en el mes de julio, por lo que el resto del año las vacunas se trasladarán a menos temperatura incrementando su tiempo de conservación.

\section{Referencias}

Álvarez, F. (2019). Capítulo 6: Transporte y conservación de las vacunas. MANUAL DE VACUNAS EN LÍNEA DE LA AEP (Asociación Española de Pediatría). Recuperado de: https://vacunasaep.org/documentos/manual/cap6\#up

Çengel, Y. A., Boles, M. A., Pedraza, C. R. C., Chávez, N. A. M., \& Cárdenas, J. L. (2006). Termodinámica (Vol. 10). São Paulo: McGrawHill.

Godoy Vaca, L. F. (2016). Diseño, construcción y evaluación energética de una cámara con celdas Peltier (efecto termoeléctrico) para refrigeración de vacunas. 130 hojas. Quito EPN. 
Valle, J., Lozano, B., Díaz, J. \& Román, R. (2019). Diseño y análisis energético de un sistema de refrigeración autónomo para el traslado de vacunas. Revista de energías renovables.

Villarrubia, M. (2001). La refrigeración termoeléctrica. Montajes e instalaciones: Revista técnica sobre la construcción e ingeniería de las instalaciones. 31(346), 65-72. 